\title{
Nanorobots in Medicine-A New Dimension in Bio Nanotechnology
}

\author{
T.Venkat Narayana Rao ${ }^{1}$, H. S. Saini ${ }^{2}$, Pinnamaneni Bhanu Prasad ${ }^{3}$ \\ ${ }^{1}$ Guru Nanak Technical Campus. Ibrahimpatanm, A.P, India. \\ ${ }^{2}$ Guru Nanak Institutions, Ibrahimpatanm, A.P, India \\ ${ }^{3}$ Guru Nanak Institutions, Ibrahimpatanm and Vision Specialist, Matrix vision GmbH, Germany \\ 1tvnrbpbby@yahoo.com
}

\begin{abstract}
Bio-nanotechnology has become a hopeful area of research that is bringing radical advancements and changes in the current century of technological mutiny. It is a one part of Nano technology. It has shown its clear participation in all fields, there is an integral part of the nanos in human science and medicine. Nanomedicine is the process of diagnosing cancers, treating, preventing disease, relieving pain, and of preserving and improving human health using molecular tools and molecular knowledge of the human body. Most of the symptoms such as fever and itching have specific biochemical reasons that can also be controlled, reduced, and eliminated using the appropriate injected nanorobots. This paper mainly concentrates on reviewing role and implementation of nanorobots in medical field and how it can replace present medical scenarios with cost reduction along with vision to permanent solutions to many human aliments towards surgery less treatments.
\end{abstract}

Keywords: Bio-nanotechnology; Nanomedicine; Nanorobots, Cancer.

\section{INTRODUCTION}

A Nanorobot is a miniature machine designed to perform a specific task with exactness of nanoscale dimensions which is precisely dimension of a few nanometers $(\mathrm{nm})$ or less. Nanobots have potential applications in the fields of assembly, maintenance and manufacturing of advanced devices, machines, circuits, at the atomic and molecular level. This process is also termed as molecular manufacturing. Nanobots have given rise to the novel field of nanomedicine. It has been suggested that a convoy of nanobots might serve as antibodies or antiviral agents in patients. There are several other potential medical applications, including repair of damaged tissue, unblocking of arteries affected by plaques. This include building complete replacement of body organs[5]. The major gain of nanobots is considered to be their durability. They can significantly perform the task of self-replication, which is the process of 
replicating themselves to replace depleted units. According to the theory, they can remain operational for years, decades, or centuries.

Nanoscale systems can also operate much faster than their larger counterparts because displacements are lesser. This allows mechanical and electrical events to happen in less time at a given speed. Nanotechnology is reasonably straightforward to understand, but developing this widespread knowledge into a nanorobot has been extra complicated. Humans are able to perform one nano-function at a time, but there are plenty of varied applications which require a construction of autonomous robot which would be exceedingly tiresome for us, irrespective of how high-tech the laboratory. So it has become very much necessary to create a whole set of specialized machine tools in order to speed the process of nanobot building[2].

\section{COMPONENTS OF NANOROBOTS}

Virtual Reality was used for the nanorobot design where is considered as a suitable approach for the use of macro and microrobotics concepts. The nanorobots are made of gold and silver colloid balls, as small as two nanometers along with carbon atoms arranged in a diamondoid structure.

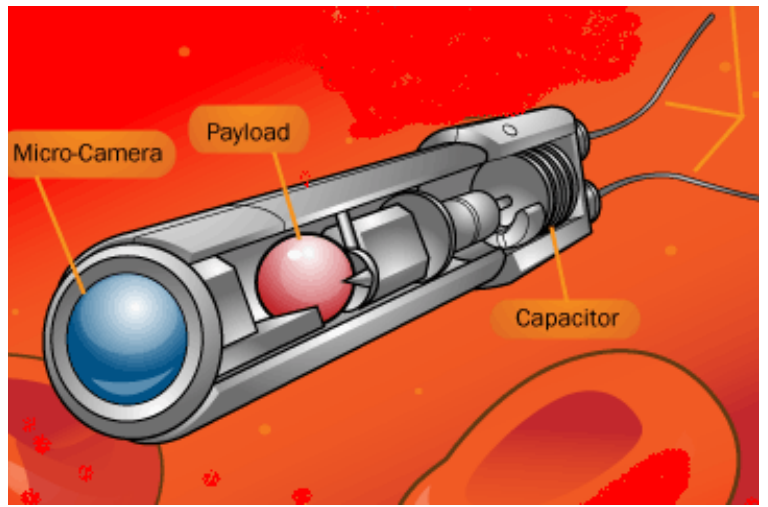

Figure 1: How Nanorobot looks like and its components.

The nanorobot design is inspired by the human body derived from biological models and is comprised by some components such as Molecular Sorting Rotor a robot arm[3]. The nanorobot movements was assumed some concepts provided from underwater robotics. The trajectories and position of each molecule, which has to be captured and assembled. Arbitrarily each one has probabilistic motion of acceleration. The nanorobot uses a macro transponder navigational system in order to address nanorobot positioning, which may keep high positional accuracy to each nanorobot's direction. Such a system might involve externally generated signal from beacons placed at fixed positions outside the skin. Thus, the delivery positions that represent organ inlets requiring proteins to be injected are located in a well-known location for the nanorobot [1].

Sensors that inform if a collision happens and identify when it is an obstacle through mircocamera, which in such cases will require a new trajectory planning, comprise the nanorobot. Plane surfaces (three fins total) and bi-directional propellers are used for the 
T.Venkat Narayana Rao, H. S. Saini and Pinnamaneni Bhanu Prasad; Nanorobots in Medicine-A New Dimension in Bio Nanotechnology, Transactions on Networks and Communications Volume 2 No 2 (2014), pp 46-57

navigation, which is comprised by two simultaneously counter-rotating screw drives for the propulsion. A binary indication is used to trigger the behavioral response as a common mechanism for action and for prevailing different phases of activity in tasks as done by social insects. In this way, activation of a motor behavior is not reliant on a specific perceptual cue, but rather on the verdict that results from sensor processing. Either touch sensors or infrared sensors can provide the information. The advantage is that the design of the motor behavior does not alter, when different sensor types of feature extraction techniques are used. Since the information needed by the motor behavior is the same binary vector in both the cases for the kinetics assumptions. The nanorobot lives in a world of adhesion, viscosity, where friction and viscous forces are dominant. Observing environmental characteristics related to nanoworlds the gravitational force here is negligible. Propellers are also extremely used to drive forward and backward. They are bi-directional and are fitted along with the propellers used. The fins that are used in the nanobots are precisely used to drive the device. The fins use plane surfaceas shown in the figure 2 . In nanobots we make essential usage of the WIFI-CMOS, hence wireless communication is feasible way to interface. It enables nanorobots in the basic operations such as tracking and diagnosis. CMOS is used for the fabrication of high performance nano devices.

\section{DESIGN OF NANOROBOTS}

There are two basic kinds of nanorobots; assemblers and self replicators. Assemblers are simple cell shaped nanorobots that are able to interpret molecules or atoms of different types, and are controlled by specific specialized programs. Self-replicators are fundamentally assemblers that are capable of duplicating themselves at a very large, fast rate; the facility to self-replicate or clone themselves, and possess subsequent ability to work in unison to build macro-scale devices[1].

\subsection{Working of Nanorobots}

The nanorobot would probably be a micron-scale robot assembled from nanoscale parts. These parts could range in size from 1-100 $\mathrm{nm}\left(1 \mathrm{~nm}=10^{-9}\right.$ meter), and might be built-in jointly to make a working machine measuring perhaps $0.5-3$ microns $\left(1\right.$ micron $=10^{-6}$ meter $)$ in diameter. The microns is about the maximum size for blood borne medical nanorobots, due to the capillary passage requirement. Carbon will likely be the chief element comprising the bulk of a medical nanorobot, most likely in the form of diamond or diamonded/fullerene nano composites largely because of the tremendous strength and chemical inertness of diamond. Many other light elements such as nitrogen, fluorine, silicon, hydrogen, sulfur, oxygen etc. will be used for special purposes in nanoscale gears and other components. It works on new concepts of novel concept of software (made up of DNAs) and hardware (made up of enzymes) molecular elements[4]. 


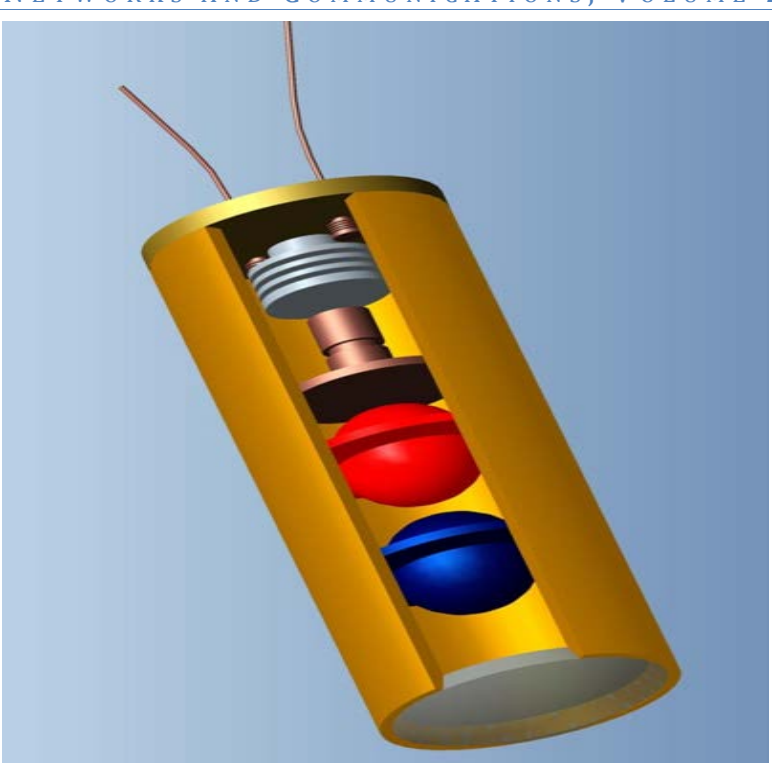

Figure 2. Woking of nanorobot with payload.

It is impossible to say precisely what a general nanorobot would look like. Nanorobots intended to travel through the bloodstream to their target will probably be 500-3000 nanometers ( 1 nanometer $=10^{-9}$ meter) in characteristic dimension. Non-blood bearing issuetraversing nanorobots might be as bulky as 50-100 microns, and alimentary or bronchial traveling nanorobots may be even larger still. Each species of medical nanorobot is to be designed to achieve a specific task, and many shapes and sizes are likely. The best nanobot consist of a transporting mechanism, an internal processor and a fuel unit that enables it to do task. The main difficulty arises around this fuel unit, since majority of usual forms of robotic propulsion cannot be minimized to nanoscale with existing technology. Scientists have succeeded in reducing a robot to five or six millimeters, but this size still technically qualifies it as a macro-robot. One possible solution is to stick on a fine film of radioactive particles to the nanobot's body. As the particles decay and release energy the nanobot would be able to harness this power source; radioactive film can be enlarged or reduced to any scale without a drop in efficiency occurring[7].

\subsection{The External Control Mechanism}

Consists of control mechanism employs affecting the dynamics of the nanorobot in its work environment through the application of external potential fields. Researchers are keenly looking at using MRI as an external control mechanism for guiding the nano particles. An MRI system is capable of generating variable magnetic field gradients which can exert force on the nanorobot in the three dimensions and control its movement and direction. Professor Martel's laboratory is exploring effect of such variable magnetic field on a ferromagnetic core that could probably be embedded into the nanorobots[1].

Other possibilities being explored are in the category of 'hybrid' control mechanisms where the target is located and fixed by an external navigational system but the behavior of the nanorobot is determined locally through an active internal control mechanism. The use of nano 
sensors and evolutionary agents to determine the nanorobots behavior is suggested by the mentioned reference.

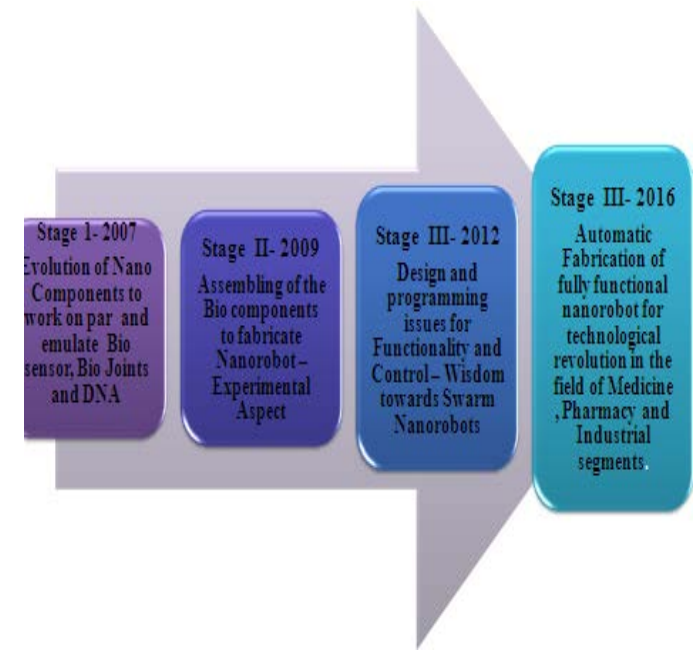

Figure 3: The Roadmap, illustrating the system capabilities improving in progression with BioNanorobotic Components with time.

\subsection{Internal Control Mechanism}

The Internal Control Mechanism is both active and passive. This type of control relies on the mechanism of bio chemical sensing and selective binding of various bio molecules with a variety of other elements. This is a traditional method, which has been in use since quite some time for designing bio molecules. Using the properties of the various bio molecules and combining with the knowledge of the target molecule that is to be influenced, these mechanisms could be valuable. However, this is a passive control mechanism where in, at run time these bio molecules cannot change their behavior. Once programmed for a particular kind of molecular interaction, these molecules stick to that. The key issues is controlling the nanorobots which are supposed to be intelligent and hence programmed and controlled to be effective in ever dynamic environment. The question of actively controlling the nanorobots using internal control mechanism is a difficult one. We require an 'active' control mechanism for the designed nanorobots such that they can vary their behavior based on situations they are subjected to, similar to the way macro robots perform. For achieving this internal control, the idea of molecular computers could be utilized. Leonard Adleman (from the University of Southern California) introduced DNA computers a decade ago to solve a mathematical problem by utilizing DNA molecules. Professor Ehud Shapiro have recently developed and has been successful in programming the biomolecular computer to analyze the biological information, which could detect and treat cancer. The molecular computer has an input and output module, that acts together, can diagnose a particular disease and in response produce a drug to cure that disease. It uses novel concept of software (made up of DNAs) and hardware (made up of enzymes) molecular elements. This molecular computer is in generalized form and can be used for any disease which produces a particular pattern of gene expression related to it[4]. 


\subsection{The Current Start of Art in Nanorobots}

Much progress has been made in the field of nanotechnology and nanomedicine, which has instigated the study of the use of robots in the nanometer scale known as nanorobots. The technology of nanorobots has grow to be a raging topic and advanced research is being carried out for the use of robots. The therapeutics of diverse fatal diseases is also in consideration for various biomedical applications and experiments in nanomedicine. The building of biosensors and the nanokinetic devices are a main requirement in the process and locomotion of nanorobots. Though, nanorobots remain to be a part of scientific fiction but this would be a clinical aspect in reality fir the future medical diagnostics. Manipulation of nanorobots is a technology that is facilitated by the Nano-Electro-Mechanical Systems or NEMS. With various new materials and structures in nanoscale, NEMS will assist in the development of new nanosensors and nanoactuators. The science of nanorobotics plays a vital role in the expansion of robots, whose structure is built by using nanoscale components and objects. The nature of the components being in the nano scale allows the researchers for the engineering of the imitation of human beings. The assembly of the various complex parts, which comprise the robots have been possible due to nanorobotics. Nanobots, nanoids, nanites, or nanomites are some of the theoretical devices created with the knowledge of nanorobotics. Development of bio-nano components from biological systems is the fore most step towards the design and development of an complex bio-nanorobot, which could be used in future applications. Since the planned systems and devices will be composed of these components, one must have a good understanding of how these perform and how could they be controlled. From the simple elements such as structural links to more advanced concepts such as motors, each component must be carefully studied and possibly manipulated to understand the practical limits of each one of them. DNA and carbon nanotubes are being fabricated into various shapes and sizes, enabling possibilities of constructing newer and complex devices. These nano-structures are potential candidates for amalgamating and accommodating the bio-nano components within them. Proteins such as rhodopsin and bacteriorhodopsin are a few examples of such bio-nano components. Both these proteins are naturally found in biological systems as light sensors. They can essentially be used as solar collectors to gather abundant energy from the sun. This energy could be produced (in terms of proton force) either for later use or could be consumed straight away by other components, such as the ATP synthase nano rotary motor. The initial work is planned to be on the bio-sensors i.e. in terms of heat shock factor. These sensors will form an integral part of the proposed bio-nano assemblies, where these will be integrated within a nano structure and will be activated, as programmed, for gathering the required information at the nano scale. The tools and techniques from molecular modeling and protein engineering will be used to design these modular components as shown in Figure 3. Bio-nanorobotics is a truly multidisciplinary field and how it has evolved step by step. DNA which may be used in a variety of ways such as a structural element and a power source, hemagglutinin virus can be used as a motor and bacteriorhodopsin could be used as a sensor or a power-source [6]. 
T.Venkat Narayana Rao, H. S. Saini and Pinnamaneni Bhanu Prasad; Nanorobots in Medicine-A New Dimension in Bio Nanotechnology, Transactions on Networks and Communications Volume 2 No 2 (2014), pp 46-57

\subsection{Bio to Bio Robotic Mapping Aspects}

This section introduces a mapping of Bio natural characteristics with that of capabilities of proposed nanorobot functionalities.

\begin{tabular}{|c|c|}
\hline $\begin{array}{l}\text { Bio Code Notation } \\
\text { and Functionality }\end{array}$ & $\begin{array}{l}\text { Capabilities Targeted Applications for the proposed fabrication of } \\
\text { Nanorobot }\end{array}$ \\
\hline $\begin{array}{l}\text { E-Energy Storage } \\
\text { and Carrier }\end{array}$ & $\begin{array}{l}\text {-Ability to store energy from various sources such as, Solar, chemical for } \\
\text { future usage and for its own working } \\
\text { - Required for the working of all the bio-chemical mechanisms of the } \\
\text { proposed bio-nano-robotic systems }\end{array}$ \\
\hline M-Mechanical & $\begin{array}{l}\text {-Ability to precisely move and orient other molecules or modules at } \\
\text { nano scale. This includes ability to mechanically bind to various target } \\
\text { objects, and carry them at desired location. } \\
\text { - Carry drugs and deliver it to the precise locations. } \\
\text { - Move micro world objects with nano precision. For example, Parallel } \\
\text { platforms for nano orientation and displacements. }\end{array}$ \\
\hline S-Sensory & $\begin{array}{l}\text {-Sensing capabilities in various domains such as, chemical, mechanical, } \\
\text { visual, auditory, electrical, magnetic } \\
\text {-Evaluation and discovery of target locations based on either chemical } \\
\text { properties, temperature or others characteristics. }\end{array}$ \\
\hline G-Signaling & $\begin{array}{l}\text { - Ability to amplify the sensory data and communicate with bio-systems } \\
\text { or with the micro controllers. } \\
\text { - Capability to identify their locations through various trigger } \\
\text { mechanisms such as fluorescence } \\
\text { - Imaging for Medical applications or for imaging changes in Nano } \\
\text { Structures }\end{array}$ \\
\hline F-Info. Storage & $\begin{array}{l}\text {-Ability to store information collected by the sensory element. Behave } \\
\text { similar to a read and write mechanism in computer field } \\
\text { - Store the sensory data for future signaling or usage } \\
\text { - Read the stored data to carry out programmed functions. } \\
\text { - Back bone for the sensory bio-module } \\
\text { - Store nano world phenomenon currently not observed with ease }\end{array}$ \\
\hline W-Swarm Behavior & $\begin{array}{l}\text { - Exhibit binding capabilities with --to perform distributive sensing, } \\
\text { intelligence actions } \\
\text {-Performed by the bio-nano robots will be planned and programmed } \\
\text { keeping in mind the swarm behavior and capabilties }\end{array}$ \\
\hline $\begin{array}{l}\text { I-Bio } \\
\text { Intelligence }\end{array}$ & $\begin{array}{l}\text {-Capability of making decisions and performing Intelligent functions } \\
\text {-Ability to make decision }\end{array}$ \\
\hline R-Replication & $\begin{array}{l}\text {-Replicate themselves when required } \\
\text { - Replicate at the target site and } \\
\text {-Replication of a particular bio-module as per the demand of the } \\
\text { situation }\end{array}$ \\
\hline
\end{tabular}




\subsection{Nanorobots in Medical Field}

The rapidly increasing field of nanotechnology has many useful and direct applications for the medical industry. The nanorobots are no exception to this rule. The medical science wants to create nanobots that can repair damaged tissue without hurt and trauma. Many of the medical procedures we employ today are very painful to the human body and do not work in agreement with our natural systems. Chemotherapy wreaks havoc on humans and nearly kills them in the pursuit to kill off their hateful cancer cells. Persistent surgical procedures are also quite common now, with associated traumas that cause several patients to die rather than healing. Nanorobots are so small that they actually relate on the same level as bacteria and viruses do, and so they are competent of blending with the very particles of our bodies like atoms and molecules. The ideal nanobot has not yet been fully fabricated, but when this microscopic robot makes its inevitable introduction it will be hailed as a lifesaver by the world of medicine. We can list out numerous uses of the nanobots in the following section[2].

\subsection{Simulation of Nanorobotic Environment}

Simulation is an indispensable tool for exploring alternatives in the configuration, motion planning and control of nanomachines exploring the human body which is practically not feasible during testing period of the proposed technology. The chief purpose of this work is to give a better understanding on the operational principle of the nanorobot through simulated environment. The technique, used will be explained with reference to the working principle of the nanorobot in the human body. The simulation approach is to enable the development of nanorobots operating in a fluid environment pertinent for medical applications. We propose a practical simulator that allows fast design methods comparing various control algorithms for nanorobots and their appropriateness for different tasks. The simulator includes identifiable targets, obstacles and thereby providing a fitting environment for a characteristic nanorobotic tasks and administrating the desired chemical/drug compositions near specific areas in human body. By the usage of simulated bio-nano environment in the ambit of virtual reality, the operator can control, design and characterize through physical simulation and 3D visualization. Based on the simulated results the real scenario of drug administration through nanrobots can be envisaged and drug testing on animals can be minimized.

\subsection{Applications of Bio-Nanorobots}

3.8.1 Microbivore nanobots: These nanobots would function similarly to the white blood cells in our bodies, but they are designed to be much faster at destroying bacteria. This type of nanobots should be able to eliminate bacterial infections in a patient within minutes, as opposed to the weeks required for antibiotics to take effect. Microbivore nanobots are designed so that antibodies attach to the particular bacteria the robot is seeking. Once bacteria attaches to an antibody, an arm grabs the bacteria and moves it to the inside of the nanorobot, 
T.Venkat Narayana Rao, H. S. Saini and Pinnamaneni Bhanu Prasad; Nanorobots in Medicine-A New Dimension in Bio Nanotechnology, Transactions on Networks and Communications Volume 2 No 2 (2014), pp 46-57

where it is destroyed. After the task the bacteria is then discharged into the bloodstream as harmless fragments.

3.8.2 Respirocyte nanobots: These nanobots would function in a similar way to the red blood cells in our bodies; however, they are designed to carry much more oxygen than natural red blood cells. This plan could be very useful for patients suffering from anemia. These reciprocate nanobots would enclose a tank in which oxygen is held at a high pressure, sensors to determine the concentration of oxygen in the bloodstream, and a valve that releases oxygen when sensors determine that additional oxygen is needed.

3.8.3 Clottocyte nanobots: These robots function likewise to the platelets in our blood. Platelets stick jointly in a wound to form a clot, preventint blood flow. Depending on the size of the wound, significant blood loss can transpire before a clot is formed A system of clottocyte nanobots would store fibers until they encounter a wound. In such case, the nanobots would disperse their fibers, which would then come closer to create a clot in a fraction of time that platelets do.

3.8.4 Cellular repair nanobots: By working at the cellular level, such nanobots could prevent much of the damage caused by comparatively clumsy blades. The nature of the components being in the nano scale allows the researchers for the engineering of the mimicking of human beings and can repair the vital body parts, similar to the natural heal process. The construction of the various complex parts, which compose the robots have been possible due to nanorobotics, nanites, nanobots, nanoids or nanomites are some of the theoretical devices created with the knowledge of nanorobotics to do such operations.

3.8.5 Nanodentistry: One of the unique applications, whereby nanorobots aid in different processes involved in dentistry. They assist in inducing oral anaesthesia, desensitization of tooth, manipulation of the tissue for the re-allignment and straightening of the irregular set of teeth $\backslash$, major tooth repair, the improvement of the teeth durability, generation of nanofiller and improvement of appearance of teeth, etc.

3.8.6 Treating arteriosclerosis: Can be applied in the therapeutics for atherosclerosis. Arteriosclerosis refers to a state, where plaque builds along the walls of arteries as shown in figure 4 . Nanorobots could conceivably treat the condition by cutting away the plaque, which would then enter into the bloodstream.

Nanorobots may treat circumstances like arteriosclerosis by physically cutting away the plaque along artery walls. 


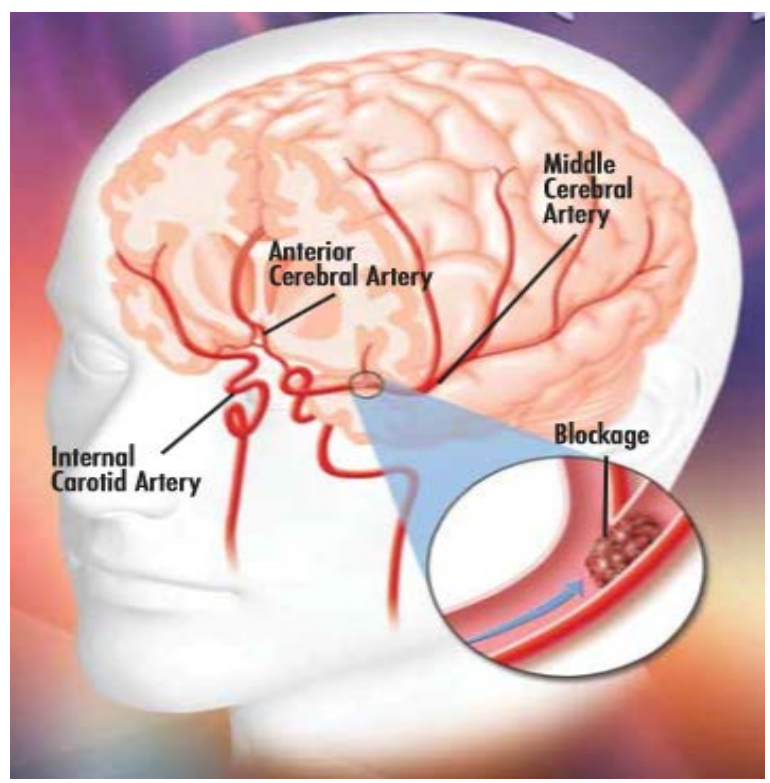

Figure 4. State of arteriosclerosis.

3.8.7 Cleaning and Breaking up blood clots: Blood clots can source complications ranging from muscle death to a stroke. Nanorobots could travel to a clot, break it up and clean as well . One particular kind of nanorobot is the clottocyte, or artificial platelet. The clottocyte carries a small mesh net that dissolves into a sticky membrane upon contact with blood plasma. This application is one of the most dangerous uses for nanorobots -- the robot must be able to remove the blockage without losing small pieces in the bloodstream, which could then travel away in the body and cause more problems. The robot must also be minute enough so that it doesn't block the flow of blood itself.

3.8.8 Parasite Removal: Nanorobots could wage micro-war on bacteria and small parasitic organisms inside a patient. It might take numerous nanorobots working together to destroy all the parasites.

3.8.9 Gout and kidney stones: Gout is a condition where the kidneys lose the ability to eradicate waste from the breakdown of fats from the bloodstream. This ruin sometimes crystallizes at points close to joints such as the ankles and knees. Patients who suffer from gout experience intense pain at these joints. A nanorobot could rupture up the crystalline structures at the joints, providing respite from the symptoms, though it would not be able to repeal the condition permanently. Kidney stones can be extremely painful, the larger the stone the more complex it is to bypass. Doctors break up large kidney stones using ultrasonic frequencies, but it's not always successful. A nanorobot could break up a kidney stones using a small laser.

Nanorobots would carry small ultrasonic signal generators to deliver frequencies directly to kidney stones. 


\section{Other related Applications}

1) Self-directed assembly: Nanorobots would have self-assembled lipidic micelles, selfassembled monolayers, and vesicles, which pursue the Brownian revolution in the medical world.

2) DNA-directed assembly: Using part of DNA for assembling, which works on the selfassembly theory of complementary base pairing and has application in the DNA based rotary motors.

3) Protein-directed assembly: Genetically engineered chaperon proteins that help in the assembly of gold nanoparticles and semiconductor quantum dots into arrays in the nanoscale range. The Ratchet action protein based molecular motors have also found much application in biology.

4) Microbes and virus directed assembly: Includes various bacteria that are included into microelectromechanical systems (MEMS) and help in acting as pumps and living motors etc. Viral capsid shells have also found application in acting as scaffolds for the assembly of the nanoparticles.

5).Transmigration of the WBC: The inflammatory cells to can be healed to accelerate the healing process.

6). Drug delivery nanorobots: Known as 'pharmacytes' will be applied in future therapeutics related to cancer in chemotherapy for exact dose administration of the chemicals as well as in the anti-HIV-therapeutics.

7). Ancillary devices: For processing different chemical reactions in the injured organs.

8). Control and monitor of glucose: In diabetic patients.

9). Surgical nanorobots for nanomanipulation: In this the target site will be subjected to programming based on the guidance from a surgeon.

10). Gene therapy and DNA analysis: For different genetic diseases and disorders.

\section{CONCLUSION}

The future of bio robots is bright. We are at the dawn of a new era in which many disciplines atreams of technology will amalgamate which include robotics, mechanical, chemical ,biomedical engineering, physics, mathematics, chemistry and biology,. This is required so as to develop fully functional systems. Research considers that the nanobots will be able to act as antibodies for patients with weak immune systems, they will be able to move in the bloodstream searching for harmful bacteria and viruses and eliminating them before they cause the patient any harm. This will become extremely useful when tackling more common ailments otherwise treating them is complex and expensive. Nanobots will not only be able to help doctors and surgeons fight life threatening diseases but also more common bacterial and viral 
cases. In this paper we have focused on the revolution of nanorobits, its functions, fabrication details along with the future research and product design avenues in the medicine field.

\section{REFERENCES}

[1]. Cavalcanti A, Freitas Robert A. Jr., Kretly Luiz C. Nanorobotics control design: a practical approach tutorial. ASME 28th Biennial Mechanisms and Robotics Conference, Salt Lake City Utah, USA, September 2004.

[2]. Zhang L, Abbott JJ, Dong L, Kratochvil BE, Bell D, Nelson BJ: Artificial bacterial flagella: fabrication and magnetic control. Appl. Physics Lett. 94 (064107), 1-3 (2009).

[3]. Ghosh A, Fischer P: Controlled propulsion of artificial magnetic nanostructured propellers. Nano Lett. 9(6), 2243-2245 (2009).

[4]. Cavalcanti A., "Assembly Automation with Evolutionary Nanorobots and Sensor-Based Control applied to Nanomedicine", IEEE Transactions on Nanotechnology, Vol. 2, no. 2, pp. 82-87, June 2003.

[5]. Venkatesan, M. ; Jolad, B. “Nanorobots in cancer treatment" , INTERACT 2010, Ladjal, H. ; Hanus, J.(L. Ferreira, A. 2009)

[6]. O. Ozcan and M. Sitti, "Modeling of Conductive Atomic Force Microscope Probes for Scanning Tunneling Microscope Operation," Micro/Nano-Letters, vol. 7, no. 4, pp. 329-333, April 2012.

[7]. Diller, C. Pawashe, S. Floyd, and M. Sitti, "Assembly and Disassembly of Magnetic Mobile Micro-Robots towards Deterministic 2-D Reconfigurable Micro-Systems," International Journal of Robotics Research, vol 31, 1667-1680, 2011. 